\title{
Revascularização do miocárdio no paciente octogenário
}

\author{
José Carlos R. IGLÉZIAS*, Luís Alberto DALLAN*, Sérgio Ferreira OLIVEIRA*, José Antônio \\ RAMIRES*, Sérgio Almeida de OLIVEIRA*, Geraldo VERGINELLI*, Fúlvio PILEGGI*, Adib D. JATENE*.
}

RBCCV 44205-123

IGLÉZIAS, J. C. R.; DALLAN, L. A.; OLIVEIRA, S. F.; RAMIRES, J. A.; OLIVEIRA, S. A.; VERGINELLI, G.; PILEGGI, F.; JATENE, A. D. - Revascularizaçāo do miocárdio no paciente octogenário. Rev. Bras. Cir. Cardiovasc., 5(3): 183-186, 1990.

RESUMO: No Instituto do Coração, de janeiro de 1978 a julho de 1990, foram tratados cirurgicamente 503 pacientes com idade igual ou superior a 80 anos. Destes, 15 foram submetidos a revascularizaçāo do miocárdio. Com o objetivo de caracterizar essa populaçāo de octogenários submetidos a revascularização do miocárdio, fizemos um levantamento de dados retrospectivamente. Neste estudo, foram analisados fatores clínicos, radiológicos, hemodinâmicos, operatórios e de pós-operatório. Nāo houve análise estatística do material. A mortalidade hospitalar foi de $2 / 15$ (13,33\%) e, num segmento médio de 24,7 meses (5-50), cinco pacientes foram a óbito devido a: acidente vascular cerebral hemorrágico, infecção do trato urinário, trombose mesentérica, infecção pulmonar e descompensaçāo diabética secundária a infecçāo do trato urinário. Dos sobreviventes, todos apresentavam melhora da sintomatologia, relacionada a angina e a insuficiência cardíaca. Baseando-nos, nos dados coletados, concluímos: 1) não houve óbito operatório; 2) a mortalidade hospitalar esteve relacionada aos processos infecciosos; 3) no seguimento tardio, a grande maioria dos pacientes referia melhora na sintomatologia e, portanto, na qualidade de vida; 4) a idade, isoladamente, náo representou fator de risco proibitivo para o tratamento cirúrgico.

DESCRITORES: miocárdio, revascularização, pacientes idosos.

\section{INTRODUÇĀO}

A revascularização do miocárdio no paciente octogenário é uma realidade em nosso meio e constitui tema de publicaçōes nacionais e internacionais com freüência crescente.

SOUZA et alii ${ }^{8}$, analisando 492 pacientes com idade igual ou superior a 70 anos, submetidos a revascularizaçāo do miocárdio isolada, ou associada a outros procedimentos, eletivas, ou em caráter de urgência, subdivididos em três subgrupos de acordo com a faixa etária, observaram que a taxa de mortalidade operatória global foi de $8 \%$ e que a mesma aumentou à medida em que a faixa etária se elevou, nos seguintes subgrupos: 70 a 74 anos, 75 a 79 anos e 80 anos em diante. A mortalidade hospitalar foi menor nos casos de revascularização isolada, eletiva quando comparada com aquela associada a outros procedimentos e esta, por sua vez, apresentava taxa menor que a dos pacientes operados em caráter de emergência, na presença de infarto agudo do miocárdio e/ou complicaçōes decorrentes dele onde a taxa de mortalidade atingiu $48 \%$.

GRONDIN et alii ${ }^{3}$, questionando a diferença de taxas de mortalidade entre pacientes com idade superior e inferior a 70 anos, submetidos a operação cardíaca, concluem que a operaçāo eletiva pode ser realizada com segurança no septuagenário, com uma expectativa para a mortalidade hospitalar menor que $5 \%$.

JOHNSON et alii ${ }^{5}$, analisando a sobrevida tar-

Trabalho realizado no Instituto do Coração do Hospital das Clínicas da Faculdade de Medicina da Universidade de Sảo Paulo. São Paulo, SP. Brasil. Recebido para publicaçáo em 8 de novembro de 1990.

* Do Instituto do Coraçáo do Hospital das Clínicas da Faculdade de Medicina da Universidade de Sáo Paulo.

Endereço para separatas: Av. Dr. Enéas de Carvalho Aguiar, 44. Divisão Cirúrgica. 05403 São Paulo, SP, Brasil. 
IGLÉZIAS, J. C. R.; DALLAN, L. A.; OLIVEIRA, S. F.; RAMIRES, J. A.; OLIVEIRA, S. A.; VERGINELLI, G.; PILEGGI, F.; JATENE, A. D. - Revascularizaçāo do miocárdio no paciente octogenário. Rev. Bras. Cir. Cardiovasc., 5(3): 183-186, 1990.

dia de pacientes submetidos a revascularização do miocárdio, entre 1972 e 1986, concluem que a idade reduz nitidamente a sobrevida para 10 a 15 anos, quando comparada com a da população em geral. Afirmam, ainda, que a disfunção ventricular esquerda severa, a operação cardíaca prévia, a doença arterial coronária difusa que requer endarterectomia afetam adversamente as taxas de sobrevida tardia. Em todos os grupos estudados, há um significativo aumento na sobrevida, quando um ou mais enxertos da artéria torácica interna são utilizados.

Com o objetivo de definir o perfil deste grupo etário, nos propusemos fazer um levantamento de dados, não somente para atualizar os existentes, mas também para obter informaçōes adicionais que nos permitissem melhorar o tratamento global oferecido.

\section{CASUÍSTICA}

Dos 503 pacientes com idade igual ou superior a 80 anos, submetidos a tratamento cirúrgico no InCór, no período analisado, 15 tiveram o miocárdio revascularizado.

Destes, dez eram do sexo feminino, 11 apresentavam até duas moléstias associadas à doença coronária, enquanto que quatro se encontravam acometidos por três ou mais moléstias associadas.

Em relação à angima do peito, ela foi considerada instável em cinco pacientes.

Quanto à classe funcional (NYHA) pré-operatória, 11 pacientes se encontravam em classe I e II e quatro em classe III e IV. Cinco pacientes não apresentavam infarto do miocárdio, no eletrocardiograma pré-operatório de repouso.

$O$ indíce cardiotorácico foi $<0,50$ em 14 pacientes.

Em relação à pressão diastólica final do ventrículo esquerdo, ela foi $\leqslant 20 \mathrm{mmHg}$ em 10 pacientes.

Onze pacientes apresentavam acometimento de três ou mais artérias coronárias.

A revascularização foi realizada eletivamente em 12 pacientes, em caráter de urgência em um e em caráter de emergência nos outros dois. Dois pacientes necessitaram de procedimentos associados: uma substituiçāo valvar e uma endarterectomia de carótida. Oito operados receberam três ou mais enxertos. Uma paciente foi revascularizada, utilizando-se a artéria torácica interna, enquanto os demais receberam somente enxertos de veia safena autógena. Quatro pacientes apresentavam acome- timento do tronco coronariano esquerdo, sendo que, em três deles, foi $\geqslant 70 \%$.

Quanto à proteção miocárdica, ela foi realizada por meio de solução cardioplégica em cinco e por pinçamento aórtico intermitente em dez pacientes.

A média de tempo para a circulação extracorpórea, foi igual a 84 minutos $(57-125)$ e a do tempo de pinçamento aórtico foi de 44,57 (20-103).

No período pós-operatório, um paciente apresentou baixo débito cardíaco, necessitando de balăo intra-aórtico, três pacientes foram acometidos por acidente vascular cerebral, um foi acometido por insuficiência renal (creatinina $>3,0 \mathrm{mg} / \mathrm{dl}$ ), quatro foram acometidos por infecçāo e dois necessitaram de assistência ventilatória por período $\geqslant 24$ horas.

A média de permanência hospitalar foi igual a 21,5 dias $(09-53)$ e os pacientes receberam alta hospitalar em média no 11,6 dia de pós-operatório (07-22). A mortalidade hospitalar foi de $2 / 15$ (13,33\%) sendo um deles devido a infecção pulmonar.

Em seguimento médio de 24,7 meses (5-50), cinco pacientes foram a óbito e suas causas determinantes foram: acidente vascular cerebral hemorrágico, infeccção do trato urinário levando a insuficiência renal crônica, trombose mesentérica, infeccçāo pulmonar e descompensaçāo diabética desencadeada por infecção do trato urinário. Dos sobreviventes, com excessão de um paciente que refere angina aos grandes esforços, os demais melhoraram a qualidade de vida, com diminuiçāo da angina e dos sintomas relacionados a insuficiência cardíaca.

Nenhuma análise estatística foi realizada, pois se trata de casuística pequena e um dos objetivos do estudo era definir o perfil deste grupo etário, submetido ao tratamento cirúrgico.

\section{RESULTADOS}

No Instituto do Coração do Hospital das Clínicas da Faculdade de medicina da Universidade de São Paulo, no período compreendido entre janeiro de 1978 e julho de 1990 , foram submetidos a revascularização do miocárdio 15 pacientes com idade igual ou superior a 80 anos.

Para a coleta de dados foi elaborado um roteiro específico e o estudo foi retrospectivo. Nele foram incluídos parâmetros clínicos, hemodinâmicos, radiológicos, operatórios e de pós-operatórios.

Baseando-se na casuística, nessa etapa do estudo observamos:

1) que não houve óbito operatório; 2) que a 
IGLÉZIAS, J. C. R.; DALLAN, L. A.; OLIVEIRA, S. F.; RAMIRES, J. A.; OLIVEIRA, S. A.; VERGINELLI, G.; PILEGGI, F.; JATENE, A. D. - Revascularização do miocárdio no paciente octogenário. Rev. Bras. Cir. Cardiovasc., 5(3): 183-186, 1990.

mortalidade hospitalar esteve relacionada aos processos infecciosos; 3) que, em seguimento tardio, a grande maioria dos pacientes refere melhora na sintomatologia e portanto na qualidade de vida e; 4) que a idade, isolamento, nāo representa um fator de risco para o tratamento cirúrgico.

\section{COMENTÁRIOS}

Concordamos com SOUZA et alii ${ }^{8}$, que, em 1990, analisando 492 pacientes com idade igual ou superior a 70 anos, submetidos a revascularizaçāo do miocárdio e subdivididos em três subgrupos de acordo com a faixa etária, observaram que a taxa de mortalidade operatória global foi igual a $8 \%$ e que a mesma aumenta à medida em que a faixa etária se eleva ( 70 a 74 anos, 75 a 79 anos e de 80 anos em diante). Em nosso levantamento, a taxa encontrada no último subgrupo foi igual $13,33 \%$.

Nossa mortalidade hospitalar foi maior que a publicada por TSAl et alii ${ }^{\circ}$, em 1989, quando da análise de pacientes septuagenários e octogenários submetidos a revascularização isolada do miocárdio com circulação extracorpórea, ao relatar mortalidade hospitalar de $6,5 \%$ nos septuagenários e $3,1 \%$ nos octogenários.

Estamos de acordo com ACINAPURA et alii ${ }^{1}$ quando, ao analisarem, comparativamente, grupos de pacientes com idade superior e inferior a 70 anos, submetidos a revascularizaçăo isolada do miocárdio, afirmam que a mortalidade é significativamente mais alta nos pacientes idosos, porém nāo está relacionada com a severidade da doença arterial coronariana, com o padrāo de angina ou com a diminuição da funçāo ventricular esquerda e que as principais causas de mortalidade foram: a falência pulmonar, renal, ambas as anteriores associadas, sepsis e complicaçőes neurológicas, Concordamos, também, com sua conclusão que os pacientes idoșos têm risco aumentado para morbidade e mortalidade, devido a causas cardíacas e nāo cardíacas, após a revascularizaçāo do miocárdio, e que o alto risco está mais associado às causas não cardíacas.

Nossa opiniāo é semelhante à de NAUNHEIN et alii $^{6}$, que analizando 23 pacientes com idade $\geqslant 80$ anos, submetidos a revascularizaçāo do miocárdio e comparados com os incluídos nos registros do estudo da cirurgia das artérias coronárias (CASS), observaram que a operação eletiva pode ser realizada no octogenário, com riscos de morbidade e mortalidade aumentados, porém aceitáveis, e que a melhora funcional a longo prazo é excelente.

Nossas observaçōes são concordantes com ENNABLI \& PELLETIER ${ }^{2}$, quando, analisando comparativamente 102 pacientes com idade $\leqslant 70$ anos contra os com idade > 70 anos, revascularizados eletivamente, observam que as complicaçōes pósoperatórias sāo cardíacas foram significativamente mais comuns $(p<0,0001)$ nos pacientes idosos $e$ que as complicações mais freqüentes foram cerebrais, esternal e respiratórias. Assim, o risco da revascularização do miocárdio está aumentado após 70 anos, particularmente devido às complicaçōes não cardíacas, que sāo responsáveis por mais da metade dos óbitos precoces, em sua casuística. Neste grupo etário (> 70 anos), é mandatório e particular atenção deve ser dada pré-operatoriamente no sentido de se evidenciarem desordens nāo cardíacas, a fim de minimizar a incidência destas complicaçōes no pós-operatório.

Nossa observação é concordante com JEFFREY et alii ${ }^{4}$, que, - em estudo de 102 homens e 102 mulheres com idade $\geqslant 70$ anos e submetidos a revascularizaçāo do miocárdio, concluíram, baseando-se na análise estatística de vários parâmetros monitorizados, que, nesta faixa etária, a operaçāo é igualmente benéfica ao homem e à mulher, em termos de sobrevida, mas que a melhora sintomática subjetiva na mulher pode ser menor.

Nossa mortalidade operatória também é maior no grupo dos octogenários e isto está concordante com ROSE et alii 7 , que, analisando 201 pacientes com idade $>70$ anos, submetidos a revascularização isolada do miocárdio comparativamente a 1242 outros pacientes com idade $<70$ anos e submetidos ao mesmo procedimento, concluíram que a mortalidade operatória foi significativamente maior no grupo dos pacientes mais idosos $(p<0,01)$. Seus dados sugerem que os pacientes idosos têm risco aumentado para morbidade e mortalidade de causas cardíacas, após revascularizaçāo do miocárdio, e que esta alta taxa de mortalidade pode resultar da falência de órgãos nāo cardíacos. 
IGLÉZIAS, J. C. R.; DALLAN, L. A.; OLIVEIRA, S. F.; RAMIRES, J. A.; OLIVEIRA, S. A.; VERGINELLI, G.; PILEGGI, F.; JATENE, A. D. - Revascularizaçāo do miocárdio no paciente octogenário. Rev. Bras. Cir. Cardiovasc., 5(3): 183-186, 1990.

RBCCV 44205-123

IGLÉZIAS, J. C. R.; DALLAN, L. A.; OLIVEIRA, S. F.; RAMIRES, J. A.; OLIVEIRA, S. A.; VERGINELLI, G.; JATENE, A. D. - Aorto-coronary bypass in elderly. Rev. Bras. Circ. Cardiovasc., 5(3): 183$186,1990$.

ABSTRACT: Five-hundred and three patients, with age equal or above 80 years, were surgically treated at the Heart Institute from January 78 to July 90 . Aiming to characterize this octogenarian population submitted to myocardial revascularization, data were retrospectivelly pursued. This study analyzed clinical,radiological, hemodynamic, operative and postoperative factors; there was no statistical analysis of the material. Hospital mortality was $2 / 15(13.33 \%)$ and in a mean follow-up of 24.7 months (5-50) five patients evoluted to death due to hemorrhagic vascular cerebral accident, urinary tract infection, mesenteric thrombosis, pulmonary infection and diabetis dysfunction, secondary to urinary tract infection. All of the survivals improved as to the sintomatology related to angina and cardiac insufficiency. Observations based on the data collected reveal that 1) there was no operative death; 2) hospital mortality was related to infectious processes; 3 ) in late follow-up the great majority of patients referred improvement as to the sintomatology and therefore in the quality of life, and 4) isolatedly, age did not represent a risk factor for surgical treatment.

DESCRIPTORS: myocardial revascularization, elderly patients.

\section{REFERÊNCIAS BIBLIOGRÁFICAS}

1 ACINAPURA, A. J.; ROSE, D. M.; CUNNINGHAN Jr., J. N.; WITZ, I. J.; KRAMER, M. D.; Zisbrod, Z. . Coronary artery bypass in septuagenarians: analysis of mortality and morbidity. Circulation, 78 (Supl. 1): $179-184,1988$.

2 ENNABLI, K. \& PELLETIER, J. - Morbidity and mortality of coronary artery surgery after the age of 70 years. Ann. Thorac. Surg., 42, 197-200, 1986.

3 GRONDIN, M. C.; THORNTON, J. C.; ENGLE J. C.; SCHREIBER, H.; CROSS, F. S. - Cardiac sugery in septuagenarians: is there a difference in mortality and morbidity. J. Thorac. Cardiovasc. Surg., 98: 908-914, 1989.

JEFFERY, D. L.; VIJAYANAGAR, R. R.; BOGNOLO, D. A.; ECKSTEIN, P. F. - Results of coronary bypass surgery in eldery women. Ann. Thorac. Surg., 42: 550-553, 1986.

5

JOHNSON, W. D.; BRENOWITZ, J. B.; KAIS, K. L. Factors influencing longterm (10 years to 15 years) survival after a sucessful coronary artery bypass operation. Ann. Thorac. Surg., 48: 19-25, 1989.
6 NAUNHEIN, K. S.; KERM, M. J.; McBRIDE, L. R.; PENNINGTON, D. G.; BARNER, H. B.; KANTER, K. R.; FIORE, A.; WILLMAN. V. L.; KAISER, G. C. Coronary artery surgery in patients aged 80 years or older. Am. J. Cardiol., 59: 804-807, 1987

7 ROSE, D. M.; GELBISH, J.; JACOBOWITZ, I. J.; KRAMER, M.; ZISBROD, Z.; ACINAPURA, A.; CAPPABIANCA, P. E.; CUNNINGHAM, J. N. Analysis of morbidity and mortality in patients 70 years of age and over undergoing isolated coronary artery bypass surgery. Am. Heart J., 110: 341 . 346, 1985.

8 SOUZA, J. M.; BERLINCK, M. F.; MOREIRA, M. G.; MARTINS, J. R. M.; MOREIRA, M. C. S.; OLIVEIRA, P. A. F.; SENNA, D. F.; OLIVEIRA, S. A. - Revascularização miocárdica em pacientes com idade igual ou superior a 70 anos (resultados imediatos e tardios 10 anos). Trabalho apresentado ao Congresso Nacional de Cirurgia Cardíaca. 17. Belo Horizonte, 1990. (Livreto de resumos, p. 38).

9 TSAI, T. P.; CHAUX, A.; KASS, R. M.; GRAY, R. J.; MATLOFF, J. M. - Aorto-coronary bypass surgery in septuagenarians and octogenarians. J. Cardiovasc. Surg., 30: 364-368, 1989. 